\title{
GENERIC RIEMANNIAN SUBMERSIONS
}

\author{
SHAHID ALI AND TANVEER FATIMA
}

\begin{abstract}
B. Sahin [12] introduced the notion of semi-invariant Riemannian submersions as a generalization of anti-invariant Riemmanian submersions [11]. As a generalization to semi-invariant Riemannian submersions we introduce the notion of generic submersion from an almost Hermitian manifold onto a Riemannian manifold and investigate the geometry of foliations which arise from the definition of a generic Riemannian submersion and find necessary and sufficient condition for total manifold to be a generic product manifold. We also find necessary and sufficient conditions for a generic submersion to be totally geodesic.
\end{abstract}

\section{Introduction}

Riemannian submersion between Riemannian manifolds was studied by B. O'Neill [10] and A. Gray [7]. Later on, such submersions have been studied widely in differential geometry. Riemannian submersions between Riemannian manifolds equipped with an additional structure of almost complex type was firstly studied by Watson [14]. Watson defined an almost Hermitian submersion between almost Hermitian manifolds and he showed that the base manifold and each fiber have the same kind of structure as the total space, in most cases. Almost Hermitian submersions have been extended to the almost contact manifolds [5], locally conformal Kaehler manifolds [9] and quaternion Kaehler manifolds [8].

Let $M$ be a complex $m$-dimensional almost Hermitian manifold with Hermitian metric $g$ and an almost complex structure $J$ and $B$ be a complex $n$-dimensional almost Hermitian manifold with metric $g_{B}$ and an almost complex structure $J^{\prime}$. A Riemannian submersion $\pi$ : $M \rightarrow B$ is called an almost Hermitian submersion if $\pi$ is an almost complex mapping, i.e., $\pi_{*} \circ J=J^{\prime} \circ \pi_{*}$. The main result of this notion is that the vertical and horizontal distributions are invariant under $J$. On the other hand, Escobales [6] studied Riemannian submersions from complex projective space onto a Riemannian manifold under the assumption that the fibres

Received July 28, 2012, accepted November 16, 2012.

Communicated by Bang-Yen Chen.

2010 Mathematics Subject Classification. 53C15, 53C40, 53C50.

Key words and phrases. Riemannian submersions, generic Riemannian submersions, totally geodesic map.

Corresponding author: Tanveer Fatima. 
are connected, complex, totally geodesic submanifolds. In fact this assumption also implies that the vertical distribution is invariant with respect to the almost complex structure.

The submersions mentioned above have one common property that the vertical and horizontal distributions are invariant. Recently B. Sahin [11] introduced the notion of antiinvariant Riemannian submersions which are Riemannian submersions from almost Hermitian manifolds such that the vertical distributions (or, for that matter the fibers) are antiinvariant under the almost complex structure of the total manifold and as a generalization of anti-invariant submersions and almost Hermitian submersions, B. Sahin introduced the notion of semi-invariant Riemannian submersion when the base manifold is an almost Hermitian manifold [12]. He has shown that such submersions are useful to investigate the geometry of the total manifold of the submersion. In the present article, we introduce the notion of a submersion from an almost Hermitian manifold under the assumption that fibers are generic submanifold of the total space and call it as a generic Riemannian submersion, and one sees it as a generalization of semi-invariant Riemannian submersions. The paper is organized as follows; In section 2 we give some basic notions of almost Hermitian manifolds and a brief introduction of Riemannian submersion between Riemannian manifolds. In section 3 , we give the definition of generic submersion and study the geometry of the distributions and their leaves. In the end of this section, we obtain decomposition theorems and find the conditions for generic submersion to be totally geodesic map.

\section{Preliminaries}

Let $(\bar{M}, g)$ be an almost Hermitian manifold. That is $\bar{M}$ admits a tensor field $J$ of type $(1,1)$ and a Riemannian metric $g$ such that

$$
J^{2}=-I, g(X, Y)=g(J X, J Y), \forall X, Y \in \Gamma(T \bar{M}) .
$$

An almost Hermitian manifold $\bar{M}$ is called Kaehler manifold if

$$
\left(\bar{\nabla}_{X} J\right) Y=0, \forall X, Y \in \Gamma(T \bar{M})
$$

where $\bar{\nabla}$ is the Levi-Civita connection on $\bar{M}$.

Let $(\bar{M}, g, J)$ be an almost Hermitian manifold and $M$ be real submanifold of $\bar{M}$, and let

$$
\mathscr{D}_{p}=T_{p} M \cap J T_{p} M, \forall p \in M
$$

such that $\mathscr{D}_{p}$ is the maximal complex subspace of $\Gamma\left(T_{p} M\right)$.

Definition 2.1 ([2]). A submanifold is said to be a $C R$-submanifold of an almost Hermitian manifold $\bar{M}$ if there exists on $M$ a $C^{\infty}$-holomorphic distribution $\mathscr{D}$ such that its orthogonal complementary distribution $\mathscr{D}^{\perp}$ is totally real i.e., $J \mathscr{D}_{p}^{\perp} \subseteq T_{p}^{\perp} M$ for all $p \in M$. A $C R$ submanifold $M$ is said to be proper if neither $\mathscr{D}=\{0\}$, nor $\mathscr{D}^{\perp}=\{0\}$. 
Now we recall the definition of slant immersion of which the complex and totally real immersions are the particular cases.

Definition 2.2 ([4]). For a non-zero vector $U$ tangent to $M$ at a point $p \in M$, the angle $\theta(U)$ between $J U$ and tangent space $T_{p} M$ is called Wirtinger angle of $U$. An immersion $f: M \rightarrow \bar{M}$ a slant immersion if the Wirtinger angle $\theta(U)$ is constant (which is independent of the choice of $p \in M$ and $U \in T_{p} M$ ).

There is yet another generalization of $C R$-submanifolds known as generic submanifolds. These submanifolds are defined by relaxing the condition on the complementary distribution to holomorphic distribution.

Definition 2.3 ([3]). A submanifold $M$ is called generic submanifold if $\operatorname{dim} \mathscr{D}_{p}$ is constant at each point $p \in M$ and $\mathscr{D}_{p}$ defines a differentiable distribution on $M$, called the holomorphic distribution.

We denote by $\mathscr{D}^{\perp}$ the orthogonal complementary distribution to $\mathscr{D}$ in $\Gamma(T M)$ and note that $J \mathscr{D}^{\perp} \cap \mathscr{D}^{\perp}=\{0\}$. We call $\mathscr{D}^{\perp}$ purely real distribution on $M$.

For any $U \in \Gamma(T M)$, we put

$$
J U=P U+F U,
$$

where $P U$ and $F U$ are the tangential and normal parts of $J U$ respectively.

Let $\Gamma\left(T^{\perp} M\right)$ be the normal bundle of $M$ in $\Gamma(T \bar{M})$. For any $N \in \Gamma\left(T^{\perp} M\right)$, we set

$$
J N=t N+f N
$$

where $t N$ and $f N$ are the tangential and normal parts of $J N$ respectively.

For generic submanifolds, we have

(i) $P \mathscr{D}=\mathscr{D}, F \mathscr{D}=\{0\}$,

(ii) $P \mathscr{D}^{\perp} \subset \mathscr{D}^{\perp}, F \mathscr{D}^{\perp} \subset T^{\perp} M$.

Let $\mu$ be the differentiable vector subbundle of $T^{\perp} M$, then by [3] we have

$$
T^{\perp} M=F \mathscr{D}^{\perp} \oplus \mu, \quad t\left(T^{\perp} M\right)=\mathscr{D}^{\perp} .
$$

Note. Throughout this article we use the definition of generic submanifold which is given by B. Y. Chen [3].

For the theory of Riemannian submersion we follow B. O'Neill [10];

Let $(M, g)$ and $\left(B, g_{B}\right)$ be Riemannian manifolds, where $\operatorname{dim}(M)=m$ and $\operatorname{dim}(B)=n$ with $m>n$. A Riemannian submersion $\pi: M \rightarrow B$ is a map from $M$ onto $B$ satisfying the following axioms; 
$\left(S_{1}\right) \pi$ has maximal rank.

$\left(S_{2}\right)$ The differential $\pi_{*}$ preserves the length of the horizontal vectors.

For each $q \in B, \pi^{-1}(q)$ is an $(m-n)$ dimensional submanifold of $M$. The submanifolds $\pi^{-1}(q), q \in B$ are called fibers. Vector fields on $M$ which are in $\left(k e r \pi_{*}\right)$ are tangent to the fibres and are called vertical vector fields; vectors which are orthogonal to the vertical distribution (or orthogonal to fibers) are said to be horizontal.

Definition 2.4. A smooth vector field $X$ on $M$ is called basic if

(i) $X$ is horizontal and

(ii) $X$ is $\pi$-related to a vector field $X_{*}$ on $B$,i.e., $\pi_{*} X_{p}=X_{* \pi(p)}$ for all $p \in M$.

We denote the projection morphisms on the distributions $\left(k e r \pi_{*}\right)$ and $\left(k e r \pi_{*}\right)^{\perp}$ by $\mathcal{V}$ and $\mathscr{H}$ respectively. Then for any $U \in \Gamma(T M)$, we put

$$
U=\mathcal{V} U+\mathscr{H} U
$$

We recall the following lemma from O’Neill [10].

Lemma 2.1. Let $\pi: M \rightarrow B$ be a Riemannian submersion between Riemannian manifolds and $X, Y$ be basic vector fields of $M$. Then we have

(a) $g(X, Y)=g_{B}\left(X_{*}, Y_{*}\right) o \pi$,

(b) the horizontal part $\mathscr{H}[X, Y]$ of $[X, Y]$ is a basic vector field and corresponds to $\left[X_{*}, Y_{*}\right]$, i.e., $\pi_{*}(\mathscr{H}[X, Y])=\left[X_{*}, Y_{*}\right]$.

(c) $[V, X]$ is vertical for any $V \in \Gamma\left(k e r \pi_{*}\right)$.

(d) $\mathscr{H}\left(\nabla_{X} Y\right)$ is the basic vector field corresponding to $\nabla_{X_{*}}^{B} Y_{*}$, where $\nabla$ and $\nabla^{B}$ are the LeviCivita connections of $g$ and $g_{B}$ respectively.

The geometry of Riemannian submersions is characterized by O'Neill's configuration tensors $\mathscr{T}$ and $\mathscr{A}$ of Riemannian submersion $\pi: M \rightarrow B$ defined for arbitrary vector fields $E$ and $F$ on $M$ by

$$
\begin{aligned}
& \mathscr{A}_{E} F=\mathscr{H} \nabla_{\mathscr{H} E} \mathcal{V} F+\mathcal{V} \nabla_{\mathscr{H} E} \not{H} F \text {, } \\
& \mathscr{T}_{E} F=\mathscr{H} \nabla_{\sqrt[V]{ } E} \mathcal{V} F+\mathscr{V} \nabla_{\mathfrak{V} E} \mathscr{H} F \text {. }
\end{aligned}
$$

It is easy to see that a Riemannian submersion $\pi: M \rightarrow B$ has totally geodesic fibers if and only if $\mathscr{T}$ vanishes identically. For any $E \in \Gamma(T M), \mathscr{T}_{E}$ and $\mathscr{A}_{E}$ are skew symmetric operators on $(\Gamma(T M), g)$ reversing the horizontal and the vertical distributions. It is also seen that $\mathscr{T}$ is 
vertical, $\mathscr{T}_{E}=\mathscr{T}_{V E}$ and $\mathscr{A}_{E}$ is horizontal, $\mathscr{A}_{E}=\mathscr{A}_{\mathcal{H E E}}$. We note that the tensor fields $\mathscr{T}$ and $\mathscr{A}$ satisfy

$$
\begin{aligned}
& \mathscr{T}_{U} W=\mathscr{T}_{W} U, \forall U, W \in \Gamma\left(\operatorname{ker} \pi_{*}\right) \\
& \mathscr{A}_{X} Y=-\mathscr{A}_{Y} X=\frac{1}{2} \mathcal{V}[X, Y], \forall X, Y \in \Gamma\left(k \operatorname{er} \pi_{*}\right)^{\perp} .
\end{aligned}
$$

On the other hand from (2.5) and (2.6) we have

$$
\begin{aligned}
\nabla_{V} W & =\mathscr{T}_{V} W+\hat{\nabla}_{V} W \\
\nabla_{V} X & =\mathscr{H}\left(\nabla_{V} X\right)+\mathscr{T}_{V} X \\
\nabla_{X} V & =\mathscr{A}_{X} V+\mathcal{V} \nabla_{X} V \\
\nabla_{X} Y & =\mathscr{H}\left(\nabla_{X} Y\right)+\mathscr{A}_{X} Y
\end{aligned}
$$

for any $X, Y \in \Gamma\left(k e r \pi_{*}\right)^{\perp}$ and $V, W \in \Gamma\left(k e r \pi_{*}\right)$, where $\hat{\nabla}_{V} W=\mathcal{V}\left(\nabla_{V} W\right)$. If $X$ is basic, then $\mathscr{H}\left(\nabla_{V} X\right)=\mathscr{A}_{X} V$.

Next, we recall the following definitions;

Definition 2.5 ([12]). A Riemannian submersion $\pi:(M, g, J) \rightarrow\left(B, g_{B}\right)$ is called a semi-invariant Riemannian submersion if there is a distribution $\mathscr{D}_{1} \subset \Gamma\left(k e r \pi_{*}\right)$ such that

$$
\left(k e r \pi_{*}\right)=\mathscr{D}_{1} \oplus \mathscr{D}_{2}, J \mathscr{D}_{1}=\mathscr{D}_{1}, J \mathscr{D}_{2} \subset\left(k e r \pi_{*}\right)^{\perp},
$$

where $\mathscr{D}_{2}$ is the orthogonal complement of $\mathscr{D}_{1}$ in $\Gamma\left(k e r \pi_{*}\right)$.

Definition 2.6 ([13]). Let $\pi$ be a Riemannian submersion from an almost Hermitian manifold $(M, g, J)$ onto a Riemannian manifold $\left(B, g_{B}\right)$. If for any non zero vector $X \in\left(k e r \pi_{*}\right)_{p}, p \in M$, the angle $\theta(X)$ between $J X$ and the space $\left(k e r \pi_{*}\right)_{p}$ is a constant, i.e., it is independent of the choice of the point $p \in M$ and choice of the vector $X$ in $\left(k e r \pi_{*}\right)_{p}$, then we say that $\pi$ is slant submersion. In this case, the angle $\theta$ is called the slant angle of the slant submersion.

Finally, we recall the notion of second fundamental form of a map between Riemannian manifolds. Let $(M, g)$ and $\left(B, g_{B}\right)$ be Riemmanian manifolds and $\phi: M \rightarrow B$ be a smooth map between them. Then the differential $\phi_{*}$ of $\phi$ can be viewed as a section of the bundle $\operatorname{Hom}\left((T M), \phi^{-1}(T B)\right) \rightarrow M$, where $\phi^{-1}(T B)$ is the pullback bundle which has fibres $\left(\phi^{-1}(T B)\right)_{p}$ $=T_{\phi(p)} B, p \in M$. Hom $\left(T M, \phi^{-1}(T B)\right)$ has a connection $\nabla$ induced from Levi-Civita connection $\nabla^{M}$ and the pull back connection. The second fundamental form $\phi$ is then given by

$$
\left(\nabla \phi_{*}\right)(X, Y)=\nabla_{X}^{\phi} \phi_{*}(Y)-\phi_{*}\left(\nabla_{X}^{M} Y\right)
$$


for any $X, Y \in \Gamma(T M)$, where $\nabla^{\phi}$ is the pullback connection. It is known that the second fundamental form is symmetric.

\section{Generic Riemannian submersions}

In this section, we define generic Riemannian submersions from an almost Hermitian manifold onto a Riemannian manifold which in fact generalizes both the semi-invariant and slant submersions, and investigate the integrability of the distributions and obtain necessary and sufficient conditions for such submersions to be totally geodesic map. We also obtain decomposition theorems for the total manifold of such submersions.

Definition 3.1. Let $M$ be a complex $m$-dimensional almost Hermitian manifold with Hermitian metric $g$ and an almost complex structure $J$ and $B$ be a Riemannian manifold with Riemannian metric $g_{B}$. A Riemannian submersion $\pi: M \rightarrow B$ is called a generic Riemannian submersion if there is a distribution $\mathscr{D}_{1} \subset \Gamma\left(k e r \pi_{*}\right)$ such that

$$
\left(\operatorname{ker} \pi_{*}\right)=\mathscr{D}_{1} \oplus \mathscr{D}_{2}, J \mathscr{D}_{1}=\mathscr{D}_{1},
$$

where $\mathscr{D}_{2}$ is the orthogonal complement of $\mathscr{D}_{1}$ in $\Gamma\left(k e r \pi_{*}\right)$, and is purely real distribution on the fibres of the submersion $\pi$.

It is known that the distribution $\left(k e r \pi_{*}\right)$ is integrable. Hence above definition implies that the integral manifold (fiber) $\pi^{-1}(q), q \in B$, of $\left(k e r \pi_{*}\right)$ is a generic submanifold of $M$. For generic submanifold we refer to [3].

For any $V \in \Gamma\left(k e r \pi_{*}\right)$ we write

$$
J V=\phi V+\omega V,
$$

where $\phi V \in \Gamma\left(\mathscr{D}_{1}\right)$ and $\omega V \in \Gamma\left(k e r \pi_{*}\right)^{\perp}$. We denote the complementary distribution to $\omega \mathscr{D}_{2}$ in $\left(\operatorname{ker} \pi_{*}\right)^{\perp}$ by $\mu$. Then we have

$$
\left(k \operatorname{er} \pi_{*}\right)^{\perp}=\omega \mathscr{D}_{2} \oplus \mu,
$$

and that $\mu$ is invariant under $J$. Thus, for any $X \in \Gamma\left(k e r \pi_{*}\right)^{\perp}$ we have

$$
J X=B X+C X
$$

where $B X \in \Gamma\left(\mathscr{D}_{2}\right)$ and $C X \in \Gamma(\mu)$.

From (3.1), (3.2) and (3.3) we have

Lemma 3.1. For a generic submersion $\pi: M \rightarrow B$, we have 
(i) $\phi \mathscr{D}_{1}=\mathscr{D}_{1}, \omega \mathscr{D}_{1}=0, \phi \mathscr{D}_{2} \subset \mathscr{D}_{2}, B\left(k e r \pi_{*}\right)^{\perp}=\mathscr{D}_{2}$,

(ii) $\phi^{2}+B \omega=-i d, C^{2}+\omega B=-i d$,

(iii) $\omega \phi+C \omega=0, B C+\phi B=0$.

We define the covariant derivative of $\phi$ and $\omega$ as follows;

$$
\begin{aligned}
& \left(\nabla_{V} \phi\right) W=\hat{\nabla}_{V} \phi W-\phi \hat{\nabla}_{V} W \\
& \left(\nabla_{V} \omega\right) W=\mathscr{H}\left(\nabla_{V} \omega W\right)-\omega \hat{\nabla}_{V} W .
\end{aligned}
$$

Then by using (2.9), (2.10), (3.1) and (3.3), we get

$$
\begin{aligned}
& \left(\nabla_{V} \phi\right) W=B \mathscr{T}_{V} W-\mathscr{T}_{V} \omega W, \\
& \left(\nabla_{V} \omega\right) W=C \mathscr{T}_{V} W-\mathscr{T}_{V} \phi W,
\end{aligned}
$$

for any $V, W \in \Gamma\left(k e r \pi_{*}\right)$.

Next, we have the following lemma;

Lemma 3.2. Let $\pi$ be generic Riemannian submersion from a Kaehler manifold $(M, g, J)$ onto a Riemannian manifold $\left(B, g_{B}\right)$. Then

(i) $\mathscr{T}_{V} \phi W+\mathscr{A}_{\omega W} V=C \mathscr{T}_{V} W+\omega \hat{\nabla}_{V} W$ and $\hat{\nabla}_{V} \phi W+\mathscr{T}_{V} \omega W=B \mathscr{T}_{V} W+\phi \hat{\nabla}_{V} W$

(ii) $\mathscr{A}_{X} B Y+\mathscr{H}\left(\nabla_{X} C Y\right)=C\left(\mathscr{H}\left(\nabla_{X} Y\right)\right)+\omega \mathscr{A}_{X} Y$ and $\mathcal{V}\left(\nabla_{X} B Y\right)+\mathscr{A}_{X} C Y=B\left(\mathscr{H}\left(\nabla_{X} Y\right)\right)+$ $\phi \mathscr{A}_{X} Y$,

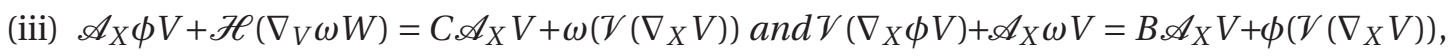
for any $X, Y \in \Gamma\left(k e r \pi_{*}\right)^{\perp}$ and $V, W \in \Gamma\left(k e r \pi_{*}\right)$.

Proof (i). Since $M$ is a Kaehler manifold, then for any $V, W \in \Gamma\left(k e r \pi_{*}\right)$ using (2.2) and (3.1) we have

$$
J \nabla_{V} W=\left\{\begin{array}{l}
\nabla_{V} J W \\
\nabla_{V} \phi W+\nabla_{V} \omega W .
\end{array}\right.
$$

Further, on using (2.9) and (2.10) we get

$$
\mathscr{T}_{V} \phi W+\hat{\nabla}_{V} \phi W+\mathscr{A}_{\omega W} V+\mathscr{T}_{V} \omega W=J\left(\mathscr{T}_{V} W+\hat{\nabla}_{V} W\right)
$$

Since $\mathscr{T}_{V} W$ and $\hat{\nabla}_{V} W$ are the horizontal and vertical, therefore again using (3.1) and (3.3), we get

$$
\mathscr{T}_{V} \phi W+\hat{\nabla}_{V} \phi W+\mathscr{A}_{\omega W} V+\mathscr{T}_{V} \omega W=B \mathscr{T}_{V} W+C \mathscr{T}_{V} W+\phi \hat{\nabla}_{V} W+\omega \hat{\nabla}_{V} W
$$

By comparing the vertical and horizontal parts in (3.4), we get the result.

Proof of $(i i)$ and $(i i i)$ follows on the similar lines as in $(i)$. 
Lemma 3.3. Let $\pi: M \rightarrow B$ be a generic Riemannian submersion from a Kaehler manifold $(M, g, J)$ onto a Riemannian manifold $\left(B, g_{B}\right)$. Then

$$
g\left(J \mathscr{T}_{U} V, \xi\right)=g\left(\mathscr{T}_{U} J V, \xi\right)
$$

for any $U \in \Gamma\left(\operatorname{ker} \pi_{*}\right), V \in \Gamma\left(\mathscr{D}_{1}\right)$ and $\xi \in \Gamma(\mu)$.

Proof. Since $M$ is a Kaehler manifold, then for any $V \in \Gamma\left(\mathscr{D}_{1}\right)$ and $U \in \Gamma\left(k e r \pi_{*}\right)$ using (2.2) we have

$$
J \nabla_{U} V=\nabla_{U} J V
$$

On using (2.9) we get

$$
J\left(\mathscr{T}_{U} V+\hat{\nabla}_{U} V\right)=\mathscr{T}_{U} J V+\hat{\nabla}_{U} J V .
$$

Taking inner product with $\xi \in \Gamma(\mu)$, we get

$$
g\left(J \mathscr{T}_{U} V, \xi\right)+g\left(J \hat{\nabla}_{U} V, \xi\right)=g\left(\mathscr{T}_{U} J V, \xi\right)+g\left(\hat{\nabla}_{U} J V, \xi\right)
$$

Since $\mu$ is invariant under $J$, then the result follows from (3.5).

Now, we investigate the integrability of the distributions $\mathscr{D}_{1}$ and $\mathscr{D}_{2}$. Since we have seen that the fibers of generic submersions from Kaehler manifolds are generic Riemannian submanifolds and $\mathscr{T}$ is the second fundamental form of the fibers, we have the following theorem;

Theorem 3.1. Let $\pi$ be a generic Riemannian submersion from a Kaehler manifold $(M, g, J)$ onto a Riemannian manifold $\left(B, g_{B}\right)$. Then the distribution $\mathscr{D}_{1}$ is integrable if and only if

$$
g\left(\mathscr{T}_{V} J W, \omega U\right)=g\left(\mathscr{T}_{W} J V, \omega U\right)
$$

for any $V, W \in \Gamma\left(\mathscr{D}_{1}\right)$ and $U \in \Gamma\left(\mathscr{D}_{2}\right)$.

Proof. Since $M$ is a Kaehler manifold, then for any $V, W \in \Gamma\left(\mathscr{D}_{1}\right),(2.2)$ and (2.9) gives

$$
\begin{aligned}
J[V, W] & =J \nabla_{V} W-J \nabla_{W} V \\
& =\nabla_{V} J W-\nabla_{W} J V \\
& =\mathscr{T}_{V} J W-\mathscr{T}_{W} J V+\hat{\nabla}_{V} J W-\hat{\nabla}_{W} J V
\end{aligned}
$$

Therefore,

$$
\mathscr{T}_{V} J W-\mathscr{T}_{W} J V=J[V, W]+\hat{\nabla}_{W} J V-\hat{\nabla}_{V} J W
$$

Now if $\mathscr{D}_{1}$ is integrable then $J[V, W] \in \Gamma\left(\mathscr{D}_{1}\right)$ as $[V, W] \in \Gamma\left(\mathscr{D}_{1}\right)$. Hence in (3.7) right hand side is vertical while the left hand side is horizontal. On comparing the horizontal and vertical part we get

$$
\mathscr{T}_{V} J W=\mathscr{T}_{W} J V
$$


for any $V, W \in \Gamma\left(\mathscr{D}_{1}\right)$. In particular, we get

$$
g\left(\mathscr{T}_{V} J W, \omega U\right)=g\left(\mathscr{T}_{W} J V, \omega U\right)
$$

Conversely, suppose if (3.7) holds, i.e.,

$$
g\left(\mathscr{T}_{V} J W-\mathscr{T}_{W} J V, \omega U\right)=0
$$

which shows that

$$
\mathscr{T}_{V} J W-\mathscr{T}_{W} J V \in \Gamma(\mu) .
$$

Now for any $\xi \in \Gamma(\mu)$, using the Lemma 3.3 and (2.7) we have

$$
g\left(\mathscr{T}_{V} J W-\mathscr{T}_{W} J V, \xi\right)=g\left(J \mathscr{T}_{V} W-J \mathscr{T}_{W} V, \xi\right)=0
$$

which implies that $\mathscr{T}_{V} J W-\mathscr{T}_{W} J V=0$, for any $V, W \in \mathscr{D}_{1}$.

Thus from (3.7), we get

$$
J[V, W]=\hat{\nabla}_{V} J W-\hat{\nabla}_{W} J V .
$$

Since $\hat{\nabla}_{V} J W-\hat{\nabla}_{W} J V$ lies in $\Gamma\left(\operatorname{ker} \pi_{*}\right)$, this implies that $[V, W]$ lies in $\Gamma\left(\mathscr{D}_{1}\right)$ and hence $\mathscr{D}_{1}$ is integrable.

Theorem 3.2. Let $\pi$ be a generic Riemannian submersion from a Kaehler manifold $(M, g, J)$ onto a Riemannian manifold $\left(B, g_{B}\right)$. Then the distribution $\mathscr{D}_{2}$ is integrable if and only if

$$
\hat{\nabla}_{W} \phi V-\hat{\nabla}_{V} \phi \omega+\mathscr{T}_{W} \omega V-\mathscr{T}_{V} \omega W \in \Gamma\left(\mathscr{D}_{2}\right),
$$

for any $V, W \in \Gamma\left(\mathscr{D}_{2}\right)$.

Proof. For any $V, W \in \gamma\left(\mathscr{D}_{2}\right)$, using (2.2), (2.9), (2.10), (3.1) and (3.3) we have

$$
\begin{aligned}
\nabla_{V} W= & -J \nabla_{V} J W \\
= & -J\left(\nabla_{V} \phi W+\nabla_{V} \omega W\right) \\
= & -J\left(\mathscr{T}_{V} \phi W+\hat{\nabla}_{V} \phi W+\mathscr{A}_{\omega W} V+\mathscr{T}_{V} \omega W\right) \\
= & -\left(B \mathscr{T}_{V} \phi W+C \mathscr{T}_{V} \phi W+\phi \hat{\nabla}_{V} \phi W+\omega \hat{\nabla}_{V} \phi W\right. \\
& \left.+B \mathscr{A}_{\omega W} V+C \mathscr{A}_{\omega W} V+\phi \mathscr{T}_{V} \omega W+\omega \mathscr{T}_{V} \omega W\right) .
\end{aligned}
$$

Similarly, we get

$$
\begin{aligned}
\nabla_{W} V= & -\left(B \mathscr{T}_{W} \phi V+C \mathscr{T}_{W} \phi V+\phi \hat{\nabla}_{W} \phi V+\omega \hat{\nabla}_{W} \phi V\right. \\
& \left.+B \mathscr{A}_{\omega V} W+C \mathscr{A}_{\omega V} W+\phi \mathscr{T}_{W} \omega V+\omega \mathscr{T}_{W} \omega V\right) .
\end{aligned}
$$


From (3.9) and (3.10), we get

$$
\begin{aligned}
{[V, W]=} & B\left(\mathscr{T}_{W} \phi V-\mathscr{T}_{V} \phi W+\mathscr{A}_{\omega V} W-A_{\omega W} V\right) \\
& +C\left(\mathscr{T}_{W} \phi V-\mathscr{T}_{V} \phi W+\mathscr{A}_{\omega V} W-A_{\omega W} V\right) \\
& +\phi\left(\hat{\nabla}_{W} \phi V-\hat{\nabla}_{V} \phi W+\mathscr{T}_{W} \omega V-\mathscr{T}_{V} \omega W\right) \\
& +\omega\left(\hat{\nabla}_{W} \phi V-\hat{\nabla}_{V} \phi W+\mathscr{T}_{W} \omega V-\mathscr{T}_{V} \omega W\right),
\end{aligned}
$$

for any $V, W \in \Gamma\left(\mathscr{D}_{1}\right) \subset \Gamma\left(\operatorname{ker} \pi_{*}\right)$. As $\left(k e r \pi_{*}\right)$ is integrable therefore $[V, W] \in \Gamma\left(k e r \pi_{*}\right)$, comparing the vertical part, we get

$$
[V, W]=B\left(\mathscr{T}_{W} \phi V-\mathscr{T}_{V} \phi W+\mathscr{A}_{\omega V} W-A_{\omega W} V\right)+\phi\left(\hat{\nabla}_{W} \phi V-\hat{\nabla}_{V} \phi W+\mathscr{T}_{W} \omega V-\mathscr{T}_{V} \omega W\right) .
$$

From (3.11) it follows that the distribution $\mathscr{D}_{2}$ is integrable if and only if

$$
\hat{\nabla}_{W} \phi V-\hat{\nabla}_{V} \phi W+\mathscr{T}_{W} \omega V-\mathscr{T}_{V} \omega W \in \Gamma\left(\mathscr{D}_{2}\right),
$$

for any $V, W \in \Gamma\left(\mathscr{D}_{2}\right)$.

For the geometry of the leaves of the distributions $\mathscr{D}_{1}$ and $\mathscr{D}_{2}$ we have the following propositions;

Proposition 3.1. Let $\pi$ be a generic Riemannian submersion from a Kaehler manifold $(M, g, J)$ onto a Riemannian manifold $\left(B, g_{B}\right)$. Then the distribution $\mathscr{D}_{1}$ defines a totally geodesic foliation if and only if

$$
\hat{\nabla}_{V_{1}} \phi W_{1} \in \Gamma\left(\mathscr{D}_{1}\right) \text { and } \mathscr{T}_{V_{1}} \phi W_{1}=0,
$$

for any $V_{1}, W_{1} \in \Gamma\left(\mathscr{D}_{1}\right)$.

Proof. For $V_{1}, W_{1} \in \Gamma\left(\mathscr{D}_{1}\right)$, using (2.2), (2.9), (3.1) and (3.3) we have

$$
\begin{aligned}
\nabla_{V_{1}} W_{1} & =-J \nabla_{V_{1}} J W_{1} \\
& =-J\left(\nabla_{V_{1}} \phi W_{1}\right) \\
& =-J\left(\mathscr{T}_{V_{1}} \phi W_{1}+\hat{\nabla}_{V_{1}} \phi W_{1}\right) \\
& =-\left(B \mathscr{T}_{V_{1}} \phi W_{1}+C \mathscr{T}_{V_{1}} \phi W_{1}+\phi \hat{\nabla}_{V_{1}} \phi W_{1}+\omega \hat{\nabla}_{V_{1}} \phi W_{1}\right)
\end{aligned}
$$

Hence $\nabla_{V_{1}} W_{1} \in \Gamma\left(\mathscr{D}_{1}\right)$ if and only if $\hat{\nabla}_{V_{1}} \phi W_{1} \in \Gamma\left(\mathscr{D}_{1}\right)$ and $\mathscr{T}_{V_{1}} \phi W_{1}=0$, which completes the proof.

Proposition 3.2. Let $\pi$ be a generic Riemannian submersion from a Kaehler manifold $(M, g, J)$ onto a Riemannian manifold $\left(B, g_{B}\right)$. Then the distribution $\mathscr{D}_{2}$ defines a totally geodesic foliation if and only if

$$
T_{V_{2}} \phi W_{2}+\mathscr{A}_{\omega W_{2}} V_{2} \in \Gamma\left(\omega \mathscr{D}_{2}\right) \text { and } \hat{\nabla}_{V_{2}} \phi W_{2}+\mathscr{T}_{V_{2}} \omega W_{2}=0
$$

for any $V_{2}, W_{2} \in \Gamma\left(\mathscr{D}_{2}\right)$. 
Proof. The proof follows from (3.9).

From Proposition 3.1 and Proposition 3.2, we have the following theorem;

Theorem 3.3. Let $\pi$ be a generic Riemannian submersion from a Kaehler manifold $(M, g, J)$ onto a Riemannian manifold $\left(B, g_{B}\right)$. Then the fibers of $\pi$ are the locally Riemannian product of leaves of $\mathscr{D}_{1}$ and $\mathscr{D}_{2}$ if and only if

$$
\begin{gathered}
\hat{\nabla}_{V_{1}} \phi W_{1} \in \Gamma\left(\mathscr{D}_{1}\right), T_{V_{1}} \phi W_{1}=0 \text {, and } \\
\mathscr{T}_{V_{2}} \phi W_{2}+\mathscr{A}_{\omega W_{2}} V_{2} \in \Gamma\left(\omega \mathscr{D}_{2}\right), \hat{\nabla}_{V_{2}} \phi W_{2}+\mathscr{T}_{V_{2}} \omega W_{2}=0,
\end{gathered}
$$

for any $V_{1}, W_{1} \in \Gamma\left(\mathscr{D}_{1}\right) V_{2}, W_{2} \in \Gamma\left(\mathscr{D}_{2}\right)$.

Now we discuss the geometry of the leaves of $\left(k e r \pi_{*}\right)$ and $\left(k e r \pi_{*}\right)^{\perp}$.

Proposition 3.3. Let $\pi: M \rightarrow B$ be generic Riemannian submersion from a Kaehler manifold $(M, g, J)$ onto a Riemannian manifold $\left(B, g_{B}\right)$. Then the distribution $\left(k e r \pi_{*}\right)^{\perp}$ defines a totally geodesic foliation if and only if

$$
\mathscr{A}_{X} B Y+\mathscr{H} \nabla_{X} C Y \in \Gamma(\mu) \text { and } \mathcal{V} \nabla_{X} B Y+\mathscr{A}_{X} C Y=0,
$$

for any $X, Y \in \Gamma\left(k e r \pi_{*}\right)^{\perp}$.

Proof. For any $X, Y \in \Gamma\left(k e r \pi_{*}\right)^{\perp}$, from (2.1) we have

$$
\nabla_{X} Y=-J \nabla_{X} J Y
$$

Then by using (3.3), (2.11) and (2.12), we get

$$
\begin{aligned}
\nabla_{X} Y= & -J\left(\nabla_{X} B Y+\nabla_{X} C Y\right) \\
= & -J\left(\left(\mathscr{A}_{X} B Y+\mathcal{V} \nabla_{X} B Y\right)+\left(\mathscr{H} \nabla_{X} C Y+\mathscr{A}_{X} C Y\right)\right) \\
= & -\left(B \mathscr{A}_{X} B Y+C \mathscr{A}_{X} B Y+\phi\left(\mathcal{V} \nabla_{X} B Y\right)+\omega\left(\mathcal{V} \nabla_{X} B Y\right)\right. \\
& \left.+B\left(\mathscr{H} \nabla_{X} C Y\right)+C\left(\mathscr{H} \nabla_{X} C Y\right)+\phi\left(\mathscr{A}_{X} C Y\right)+\omega\left(\mathscr{A}_{X} C Y\right)\right)
\end{aligned}
$$

From (3.12) it follows that $\left(\operatorname{ker} \pi_{*}\right)^{\perp}$ defines a totally geodesic foliation if and only if

$$
B\left(\mathscr{A}_{X} B Y+\mathscr{H} \nabla_{X} C Y\right)+\phi\left(\mathcal{V} \nabla_{X} B Y+\mathscr{A}_{X} C Y\right)=0 .
$$

Which then yields

$$
\begin{aligned}
B\left(\mathscr{A}_{X} B Y+\mathscr{H} \nabla_{X} C Y\right) & =0 \\
\phi\left(\mathcal{V} \nabla_{X} B Y+\mathscr{A}_{X} C Y\right) & =0 .
\end{aligned}
$$

Hence the result.

For the distribution $\left(\operatorname{ker} \pi_{*}\right)$, we have 
Proposition 3.4. Let $\pi$ be a generic Riemannian submersion from a Kaehler manifold $(M, g, J)$ onto a Riemannian manifold $\left(B, g_{B}\right)$. Then the distribution $\left(k e r \pi_{*}\right)$ defines a totally geodesic foliation if and only if

$$
T_{V} \phi W+\mathscr{A}_{\omega W} V \in \Gamma\left(\omega \mathscr{D}_{2}\right) \text { and } \hat{\nabla}_{V} \phi W+\mathscr{T}_{V} \omega W \in \Gamma\left(\mathscr{D}_{1}\right),
$$

for any $V, W \in \Gamma\left(k e r \pi_{*}\right)$.

Proof. For any $V, W \in \Gamma\left(k e r \pi_{*}\right)$ using (2.2), (2.9), (2.10) and (3.1) we get

$$
\begin{aligned}
\nabla_{V} W= & -J \nabla_{V} J W \\
= & -J\left(\nabla_{V} \phi \omega+\nabla_{V} \omega W\right) \\
= & -J\left(\mathscr{T}_{V} \phi W+\hat{\nabla}_{V} \phi W+\mathscr{A}_{\omega W} V+\mathscr{T}_{V} \omega W\right) \\
= & -\left(B \mathscr{T}_{V} \phi W+C \mathscr{T}_{V} \phi W+\phi \hat{\nabla}_{V} \phi W+\omega \hat{\nabla}_{V} \phi W\right. \\
& \left.+B \mathscr{A}_{\omega W} V+C \mathscr{A}_{\omega W} V+\phi \mathscr{T}_{V} \omega W+\omega \mathscr{T}_{V} \omega W\right) \\
\nabla_{V} W= & -B\left(\mathscr{T}_{V} \phi W+\mathscr{A}_{\omega W} V\right)-\phi\left(\hat{\nabla}_{V} \phi W+\mathcal{V} \nabla_{V} \omega W\right) \\
& -C\left(\mathscr{T}_{V} \phi W+\mathscr{A}_{\omega W} V\right)-\omega\left(\hat{\nabla}_{V} \phi W+\mathcal{V} \nabla_{V} \omega W\right)
\end{aligned}
$$

From above equation, it follows that $\left(\operatorname{ker} \pi_{*}\right)$ defines a totally geodesic foliation if and only if

$$
C\left(\mathscr{T}_{V} \phi W+\mathscr{A}_{\omega W} V\right)+\omega\left(\hat{\nabla}_{V} \phi W+\mathcal{V} \nabla_{V} \omega W\right)=0
$$

which implies

$$
\mathscr{T}_{V} \phi W+\mathscr{A}_{\omega W} V \in \Gamma\left(\omega \mathscr{D}_{2}\right) \text { and } \hat{\nabla}_{V} \phi W+\mathcal{V} \nabla_{V} \omega W \in \Gamma\left(\mathscr{D}_{1}\right)
$$

From Theorem 3.3 and Proposition 3.3, we have the following decomposition for total space;

Theorem 3.4. Let $\pi$ be a generic Riemannian submersion from a Kaehler manifold $(M, g, J)$ onto a Riemannian manifold $\left(B, g_{B}\right)$. Then the total space $M$ is a generic product manifold of the leaves of $\mathscr{D}_{1}, \mathscr{D}_{2}$ and $\left(k e r \pi_{*}\right)^{\perp}$, i.e., $M=M_{\mathscr{D}_{1}} \times M_{\mathscr{D}_{2}} \times M_{\left(k e r \pi_{*}\right)}$, if and only if

$$
\begin{array}{r}
\hat{\nabla}_{V_{1}} \phi W_{1} \in \Gamma\left(\mathscr{D}_{1}\right), T_{V_{1}} \phi W_{1}=0, \\
\mathscr{T}_{V_{2}} \phi W_{2}+\mathscr{A}_{\omega W_{2}} V_{2} \in \Gamma\left(\omega \mathscr{D}_{2}\right), \hat{\nabla}_{V_{2}} \phi W_{2}+\mathscr{T}_{V_{2}} \omega W_{2}=0
\end{array}
$$

and

$$
\mathscr{A}_{X} B Y+\mathscr{H} \nabla_{X} C Y \in \Gamma(\mu), \mathcal{V} \nabla_{X} B Y+\mathscr{A}_{X} C Y=0,
$$

for any $V_{1}, W_{1} \in \Gamma\left(\mathscr{D}_{1}\right), V_{2}, W_{2} \in \Gamma\left(\mathscr{D}_{2}\right)$, and $X, Y \in \Gamma\left(k e r \pi_{*}\right)^{\perp}$, where $M_{\mathscr{D}_{1}}, M_{\mathscr{D}_{2}}$ and $M_{\left(k e r \pi_{*}\right)^{\perp}}$ are leaves of the distributions $\mathscr{D}_{1}, \mathscr{D}_{2}$ and $\left(k e r \pi_{*}\right)^{\perp}$ respectively. 
From Proposition 3.3 and Proposition 3.4, we have

Theorem 3.5. Let $\pi$ be a generic Riemannian submersion from a Kaehler manifold $(M, g, J)$ onto a Riemannian manifold $\left(B, g_{B}\right)$. Then $M$ is generic product manifold if and only if

$$
\mathscr{A}_{X} B Y+\mathscr{H} \nabla_{X} C Y \in \Gamma(\mu), \mathcal{V} \nabla_{X} B Y+\mathscr{A}_{X} C Y=0
$$

and

$$
\mathscr{T}_{V} \phi W+\mathscr{A}_{\omega W} V \in \Gamma\left(\omega \mathscr{D}_{2}\right), \hat{\nabla}_{V} \phi W+\mathcal{V} \nabla_{V} \omega W \in \Gamma\left(\mathscr{D}_{1}\right)
$$

for any $X, Y \in \Gamma\left(k e r \pi_{*}\right)^{\perp}$ and $V, W \in \Gamma\left(k e r \pi_{*}\right)$.

Now we obtain necessary and sufficient condition for generic Riemannian submersion to be totally geodesic. We recall that a differential map $\pi$ between Riemannian manifolds $(M, g)$ and $(B, B)$ is called totally geodesic map if

$$
\left(\nabla \pi_{*}\right)(X, Y)=0 \text {, for all } X, Y \in \Gamma(T M) \text {. }
$$

Theorem 3.6. Let $\pi$ be a generic Riemannian submersion from a Kaehler manifold $(M, g, J)$ onto a Riemannian manifold $\left(B, g_{B}\right)$. Then $\pi$ is a totally geodesic map if and only if

$$
\begin{aligned}
& \hat{\nabla}_{V} \phi W+\mathscr{T}_{V} \omega W \in \Gamma\left(\mathscr{D}_{1}\right), \\
& \mathscr{T}_{V} \phi W+\mathscr{A}_{\omega W} V \in \Gamma\left(\omega \mathscr{D}_{2}\right), \\
& \hat{\nabla}_{V} B X+\mathscr{T}_{V} C X \in \Gamma\left(\mathscr{D}_{1}\right) \text { and } \\
& \mathscr{T}_{V} B X+\mathscr{A}_{C X} V \in \Gamma\left(\omega_{2}\right),
\end{aligned}
$$

for any $X \in \Gamma\left(k e r \pi_{*}\right)^{\perp}$ and $V, W \in \Gamma\left(k e r \pi_{*}\right)$

Proof. Since $\pi$ is a Riemannian submersion, we have

$$
\left(\nabla \pi_{*}\right)(X, Y)=0, \text { for all } X, Y \in \Gamma\left(k e r \pi_{*}\right)^{\perp} .
$$

For any $V, W \in \Gamma\left(\operatorname{ker}_{*}\right)$ using (2.2), (2.9), (2.10), (2.13) and (3.1), we get

$$
\begin{aligned}
\left(\nabla \pi_{*}\right)(V, W)= & -\pi_{*}\left(\nabla_{V} W\right) \\
= & -\pi_{*}\left(-J \nabla_{V} J W\right) \\
= & \pi_{*}\left(J \nabla_{V}(\phi W+\omega W)\right) \\
= & \pi_{*}\left(J\left(\mathscr{T}_{V} \phi W+\hat{\nabla}_{V} \phi W\right)+J\left(\mathscr{A}_{\omega W} V+\mathscr{T}_{V} \omega W\right)\right) \\
= & \pi_{*}\left(\left(B \mathscr{T}_{V} \phi W+C \mathscr{T}_{V} \phi W\right)+\left(\phi \hat{\nabla}_{V} \phi W+\omega \hat{\nabla}_{V} \phi W\right)\right. \\
& \left.+\left(B \mathscr{A}_{\omega W} V+C \mathscr{A}_{\omega W} V\right)+\left(\phi \mathscr{T}_{V} \omega W+\omega \mathscr{T}_{V} \omega W\right)\right) .
\end{aligned}
$$


Thus $\left(\nabla \pi_{*}\right)(V, W)=0$ if and only if

$$
\omega\left(\hat{\nabla}_{V} \phi W+\mathscr{T}_{V} \omega W\right)+C\left(\mathscr{T}_{V} \phi W+\mathscr{A}_{\omega W} V\right)=0
$$

On the other hand using (2.2), (2.9), (2.10) and (3.3) for any $X \in \Gamma\left(k e r \pi_{*}\right)^{\perp}$ and $V \in$ $\Gamma\left(\operatorname{ker} \pi_{*}\right)$, we get

$$
\begin{aligned}
\left(\nabla \pi_{*}\right)(V, X)= & -\pi_{*}\left(\nabla_{V} X\right) \\
= & -\pi_{*}\left(-J \nabla_{V} J X\right) \\
= & \pi_{*}\left(J\left(\nabla_{V} B X+\nabla_{V} C X\right)\right) \\
= & \pi_{*}\left(\left(B \mathscr{T}_{V} B X+C \mathscr{T}_{V} B X\right)+\left(\phi \hat{\nabla}_{V} B X+\omega \hat{\nabla}_{V} B X\right)\right. \\
& \left.+\left(B \mathscr{A}_{C X} V+C \mathscr{A}_{C X} V\right)+\left(\phi \mathscr{T}_{V} C X+\omega \mathscr{T}_{V} C X\right)\right)
\end{aligned}
$$

Thus $\left(\nabla \pi_{*}\right)(V, X)=0$ if and only if

$$
\omega\left(\hat{\nabla}_{V} B X+\mathscr{T}_{V} C X\right)+C\left(\mathscr{T}_{V} B X+\mathscr{A}_{C X} V\right)=0
$$

The result then follows from (3.14) and (3.15) and (3.16).

Now we recall that a Riemannian submersion from a Riemannian manifolds $(M, g)$ onto a Riemannian manifold $\left(B, g_{B}\right)$ is called a Riemannian submersion with totally umbilical fibres if

$$
\mathscr{T}_{V} W=g(V, W) H
$$

for $V, W \in \Gamma\left(k e r \pi_{*}\right)$, where $H$ is mean curvature vector field of the fibres [12].

We then have

Proposition 3.5. Let $\pi$ be a generic Riemannian subersion with totally umbilical fibres from a Kaehler manifold $(M, g, J)$ onto a Riemannian manifold $\left(B, g_{B}\right)$, then $H \in \Gamma\left(\omega \mathscr{D}_{2}\right)$.

Proof. From (2.2), we have

$$
\nabla_{V} J W=J \nabla_{V} W
$$

for any $V, W \in \Gamma\left(\mathscr{D}_{1}\right)$. Now using (3.1) and (3.3) we obtain

$$
\begin{aligned}
\mathscr{T}_{V} J W+\hat{\nabla}_{V} J W & =J\left(\mathscr{T}_{V} W+\hat{\nabla}_{V} W\right) \\
& =B \mathscr{T}_{V} W+C \mathscr{T}_{V} W+\phi \hat{\nabla}_{V} W+\omega \hat{\nabla}_{V} W .
\end{aligned}
$$

Taking inner product in (3.18) with $X \in \Gamma(\mu)$ and then using (3.17) we get

$$
\begin{aligned}
g\left(\mathscr{T}_{V} J W, X\right) & =g\left(C \mathscr{T}_{V} W, X\right), \\
g(V, J W) g(H, X) & =g\left(J \mathscr{T}_{V} W, X\right),
\end{aligned}
$$




$$
\begin{aligned}
& g(V, J W) g(H, X)=g\left(J \mathscr{T}_{V} W, J X\right), \\
& g(V, J W) g(H, X)=-g(V, W) g(H, J X)
\end{aligned}
$$

Interchanging $V$ and $W$ in (3.19), we get

$$
g(W, J V) g(H, X)=-g(V, W) g(H, J X)
$$

Combining (3.19) and (3.20) we get $g(H, J X)=0$ which shows that $H \in \Gamma\left(\omega \mathscr{D}_{2}\right)$.

\section{References}

[1] P. Baird and J. C. Wood, Harmonic morphisms between Riemannian manifolds, London Mathematical Society, Monographs, 29, Oxford University Press 2003.

[2] A. Bejancu, CR-submanifolds of Kaehler manifolds I, Proc. Amer. Math. Soc., 69(1978), 134-142.

[3] B. Y. Chen, Differential geometry of real submanifolds in a Kaehler manifold, Monatsh. Math., 91 (1981), 257274.

[4] B. Y. Chen, Geometry of slant submanifods, Katholieke Universiteit Leuven, Leuven, 1990.

[5] D. Chinea, Almost contact metric submersions, Rand, Circ. Math. Palermo, 34(1985), 89-104.

[6] R. H. Jr. Escobales, Riemannian submersions from complex projective spaces, J. Differential Geometry, 13(1978), 93-107.

[7] A. Gray, Pseudo-Riemannian almost product manifolds and submersions, J. Math. Mich., 16(1967), 715-737.

[8] S. Ianus, R. Mazzocco and G. E. Vilice, Riemannian submersions from quaternion manifolds, Acta. Appl. Math., 104(2008), 83-89.

[9] J. C. Marreroand and J. Rocha, Locally conformal Kaehler submersions, Geom. Dedicata, 53(1994), $271-289$.

[10] B. O'Neill, The fundamental equations of a submersion, Mich. Math. J., 13(1966), 458-469.

[11] B. Sahin, Anti-invariant Riemannian submersions from almost Hermitian manifolds, 8 Central European J. math, No. 3 (2010), 437-447.

[12] B. Sahin, Semi-invariant submersions from almost Hermitian manifolds, Canad. Math. Bull, 54(2011).

[13] B. Sahin, Slant submersions from almost Hermitian manifolds, Bull. Math. Soc. Sci. Math. Roumanie, 1(2011), 93-105.

[14] B. Watson, Almost Hermitian submersions, J. Differential Geometry, 11(1976), 147-165.

[15] K. Yano and M. Kon, Structures on manifolds, World Scientific, Singapore, 1984.

[16] K. Yano and M. Kon, Generic submanifolds, Ann. Mat. Pura App., 123(1980), 59-92.

[17] K. Yano and M. Kon, Generic submanifolds of sasakian manifolds, Kodai Math. J., 3(1980), $163-196$.

Department of Mathematics, Aligarh Muslim University, Aligarh 202002, India.

E-mail: shahid07ali@gmail.com

Department of Mathematics, Aligarh Muslim University, Aligarh 202002, India.

E-mail: fatima.tanveer.maths@gmail.com 